\title{
Identifying the Dynamic Model Used by the KUKA LWR: A Reverse Engineering Approach
}

\author{
Claudio Gaz Fabrizio Flacco Alessandro De Luca
}

\begin{abstract}
An approach is presented for the model identification of the so-called link dynamics used by the KUKA LWRIV, a lightweight manipulator with elastic joints that is very popular in robotics research but for which a complete and reliable dynamic model is not yet publicly available. The control software interface of this robot provides numerical values of the link inertia matrix and the gravity vector at each configuration, together with link position and joint torque sensor data. Taking advantage of this information, a general procedure is set up for determining the structure and identifying the value of the relevant dynamic coefficients used by the manufacturer in the evaluation of these robot model terms. We call this a reverse engineering approach, because our main goal is to match the numerical data provided by the software interface, using a suitable symbolic model of the robot dynamics and the inertial and gravity coefficients that are being estimated. Only configuration-dependent terms are involved in this process, and thus static experiments are sufficient for this task. The main issues of dynamic model identification for robots with elastic joints are discussed in general, highlighting the pros and cons of the approach taken for this class of KUKA lightweight manipulators. The main identification results, including training and validation tests, are reported together with additional dynamic validation experiments that use the complete identified model and joint torque sensor data.
\end{abstract}

\section{INTRODUCTION}

The development of complete and accurate dynamic models of robot manipulators is a necessary step for the design of motion controllers with superior performance [1]. Starting from the historical works on the Unimation Puma robot [2] in the early days of robotics research, several methods and procedures for the experimental identification of dynamic models of industrial robots have been formally developed [3], [4], and the main techniques have reached by now a full maturity [5].

In the recent years, a large interest has been devoted to a new generation of lightweight manipulators intended for safer physical human-robot interaction and improved energy efficiency. Laboratory or specialized prototypes, such as the WAMS by Salisbury [6] and the lightweight series at DLR [7], have led now to commercial robots for research, service, and industrial applications, with the Barrett manipulator [8] and the KUKA LWR-IV arm [9] as two major representative products.

A common characteristics of these robots is that they intentionally include compliance at the joints, due to the presence of harmonic drives or flexible transmission cables. This

The authors are with the Dipartimento di Ingegneria Informatica, Automatica e Gestionale, Sapienza Università di Roma, Via Ariosto 25, 00185 Roma, Italy (\{gaz,fflacco,deluca\}@diag.uniroma1.it). This work is supported by the European Commission, within the FP7 ICT-287513 SAPHARI project (www.saphari.eu). poses some extra challenges to the dynamic modeling and its parametric identification, because of the extra variables and dynamic terms involved, the more complex equations, and the need of additional sensors [10]. Extensions of the identification methods that work for rigid robots are possible in order to cover the model of robots with elastic joints, including link and rotor inertias as well as joint stiffness. However, complete and satisfactory results are not available at present, at least to the authors' knowledge.

While customers of the Barrett manipulator have already access to the details of the dynamic model and numerical parameters of this arm, as provided by the designer/manufacturer, KUKA has not yet released a public version of the dynamic model of its lightweight robot. Nonetheless, the control software of the LWR, in particular its Fast Research Interface (FRI), can provide to the end-user the numerical values of the elements of the link inertia matrix and of the gravity vector at the current robot configuration (apparently, evaluated by a Newton-Euler routine). Since the current link position and joint torque sensor data are also available at a fast rate, this opens the way to an alternative identification procedure of dynamic model terms. The main goal of this paper is to show if and how, with a suitable symbolic modeling of the KUKA LWR-IV dynamics, we can perform an off-line identification of the unknown gravity and inertial coefficients based only on the numerical data provided by the software interface.

We shall see that a series of link position measurements and data reading collected in different static configurations (and not necessarily along exciting motion trajectories ${ }^{1}$ ) allows a very accurate estimation of the numerical values of the dynamic coefficients used by the robot manufacturer. Using a symbolic dynamic model of the robot link dynamics obtained in a classical way [1] and the inertial dynamic coefficients obtained with this sort of 'reverse engineering' procedure, it is rather straightforward to derive also Coriolis and centrifugal terms. Validation of this dynamic identification process can be performed in a more traditional way, letting the robot move in its workspace and comparing the torques predicted by the identified model with the measurements coming from the joint torque sensors. It should be emphasized that the possibility of separating the link dynamics from the motor dynamics, so as to perform identification only of the former,

\footnotetext{
${ }^{1}$ Actually, it is possible to retrieve the same data readings during robot motion. The choice of using static and randomly extracted positions has been carried out in order to maximize the information content of the data. We have repeated also our tests with the robot motion without finding sensible differences.
} 
is a direct consequence of the presence of joint compliance and elastic torque measurements.

The paper is organized as follows. After a brief summary on the KUKA LWR system (Sect. II), the issues of dynamic identification for robots with elastic joints are discussed in Sect. III. The assumptions made for developing the dynamic model in symbolic form and the actual identification methodology are described in Sects. IV and V, respectively. Section VI reports the obtained numerical results and their discussion.

\section{KUKA LWR RoвOT}

The KUKA LWR robot (Light Weight Robot), in particular in its release $\mathrm{IV}+$ considered in this paper, is characterized by an extremely light anthropomorphic structure with 7 revolute joints, driven by compact brushless motors via harmonic drives. The presence of such transmission elements introduces a dynamically time-varying elastic displacement at each joint, between the angular position of the motor and that of the driven link. The total weight is approximately $16 \mathrm{~kg}$, with a rated payload of $7 \mathrm{~kg}$. All joints are equipped with position sensors on the motor and link sides, and with a joint torque sensor.

Figure 1 shows the robot in its zero position, together with the link frames chosen according to the Denavit-Hartenberg convention. The associated parameters are given in Tab. I. For the purpose of dynamic analysis, the origins of the base (frame 0) and end-effector (frame 7) frames are taken coincident respectively with the origins of the first and sixth link frames. In this way, only the two link lengths $d_{1}$ and $d_{2}$ are left as non-zero kinematic parameters.

The KR C2 1r robot controller unit, together with the socalled Fast Research Interface (FRI) [11], is able to provide (at a 1 msec sampling rate) the link position $\boldsymbol{q}$ and joint torque $\tau_{J}$ measurements, as well as the numerical values of the link inertia matrix $\boldsymbol{M}_{\text {num }}(\boldsymbol{q})$, of the gravity vector $\boldsymbol{g}_{\text {num }}(\boldsymbol{q})$, and of the robot Jacobian matrix $\boldsymbol{J}_{\text {num }}(\boldsymbol{q})$ at the

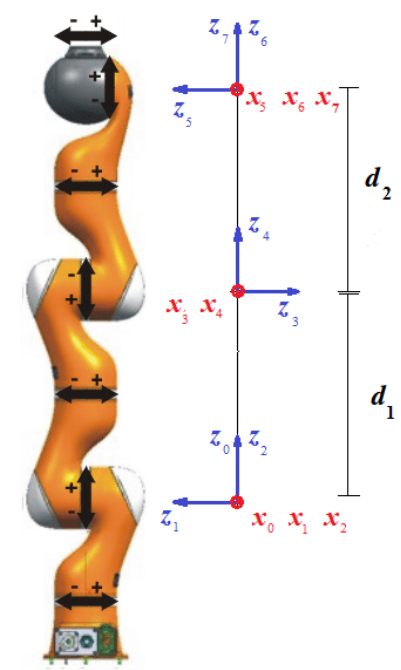

Fig. 1. Denavit-Hartenberg frames of the KUKA LWR-IV: All $x$-axes point toward the viewer (frames are displaced sideways for better clarity)
TABLE I

DENAVIT-HARTENBERG PARAMETERS OF THE KUKA LWR-IV

\begin{tabular}{ccrll}
\hline Link & $a_{i}$ & $\alpha_{i}$ & $d_{i}$ & $\theta_{i}$ \\
\hline 1 & 0 & $\pi / 2$ & 0 & $q_{1}$ \\
2 & 0 & $-\pi / 2$ & 0 & $q_{2}$ \\
3 & 0 & $-\pi / 2$ & $d_{1}$ & $q_{3}$ \\
4 & 0 & $\pi / 2$ & 0 & $q_{4}$ \\
5 & 0 & $\pi / 2$ & $d_{2}$ & $q_{5}$ \\
6 & 0 & $-\pi / 2$ & 0 & $q_{6}$ \\
7 & 0 & 0 & 0 & $q_{7}$ \\
\hline
\end{tabular}

current configuration (Fig. 2). The controller is designed in such a way that the command $\boldsymbol{u}_{\text {user }}$ given by the user, which can be in particular a desired joint position $\boldsymbol{q}_{\mathrm{d}}$ or velocity $\dot{\boldsymbol{q}}_{\mathrm{d}}$, or a user specified joint torque $\tau_{\text {user }}$ as commanded through the FRI library, is conveniently manipulated so as to have the motors generate the appropriate torque $\tau$ for the commanded task. Moreover, the FRI software library is at disposal of the scientific community [12], supplying many useful tools for robot communication and implementations of joint and Cartesian position or impedance controllers.

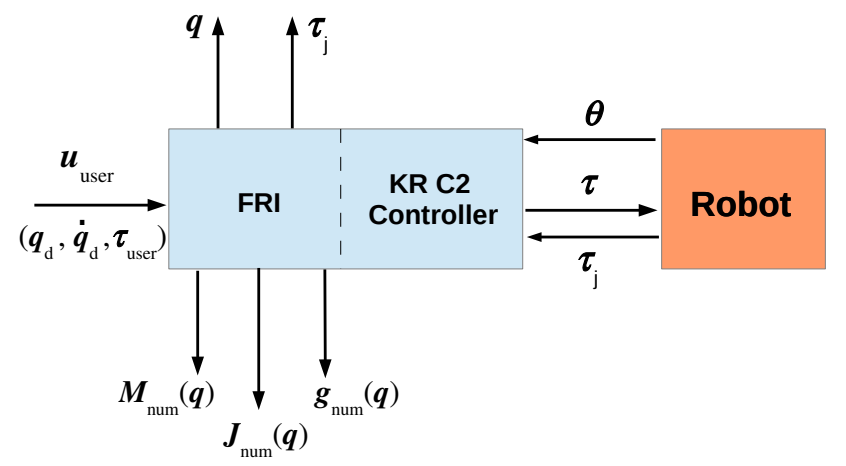

Fig. 2. Signal flows from/to the KUKA LWR robot and the KR C2 controller

\section{DYNAMIC IDENTIFICATION OF RoBOTS WITH ELASTIC JOINTS}

For a robot with elastic joints, let $\boldsymbol{\theta}$ be the $n$-vector of motor (i.e., rotor) positions, as reflected through the reduction gears, and $\boldsymbol{q}$ the $n$-vector of link positions. Under the standard modeling assumptions introduced by Spong [13], the dynamic model takes the form

$$
\begin{aligned}
\boldsymbol{M}(\boldsymbol{q}) \ddot{\boldsymbol{q}}+\boldsymbol{c}(\boldsymbol{q}, \dot{\boldsymbol{q}})+\boldsymbol{g}(\boldsymbol{q}) & =\boldsymbol{K}(\boldsymbol{\theta}-\boldsymbol{q}) \\
\boldsymbol{B} \ddot{\boldsymbol{\theta}}+\boldsymbol{K}(\boldsymbol{\theta}-\boldsymbol{q}) & =\boldsymbol{\tau}-\boldsymbol{\tau}_{F}
\end{aligned}
$$

where $M$ is the positive definite, symmetric inertia matrix of the robot links (including the motor masses), $c$ is the vector of Coriolis and centrifugal terms (quadratic in $\dot{\boldsymbol{q}}$ ), $\boldsymbol{g}$ is gravitational vector, $\boldsymbol{B}$ is the positive definite, diagonal matrix of motor inertias (reflected through the gear ratios), $\boldsymbol{K}$ is the positive definite, diagonal matrix of joint stiffness, $\tau$ are the motor torques, and $\boldsymbol{\tau}_{F}$ are dissipative torques acting on the motor side of the transmissions. The two $n$-dimensional 
second-order differential equations (1) and (2) are referred to as the link and the motor equations, respectively.

It should be noted that all dynamic terms depend at most on link variables only, that friction effects can be neglected in the link equations (this is especially true for the DLR LWRIII and the KUKA LWR-IV robots), and that the link and motor equations are coupled only through the elastic torque

$$
\tau_{J}=\boldsymbol{K}(\boldsymbol{\theta}-\boldsymbol{q})
$$

This is also the quantity measured by joint torque sensors. These properties are all relevant for setting up a specific dynamic identification procedure.

Model identification methods for rigid robots rely heavily on the linear parametrization property of their link dynamics [1], namely on the fact that the left-hand side of eq. (1) can be factorized as $\boldsymbol{Y}_{R}(\boldsymbol{q}, \dot{\boldsymbol{q}}, \ddot{\boldsymbol{q}}) \boldsymbol{\pi}_{R}$, where the subscript $R$ refer the $\left(n \times p_{R}\right)$ regressor matrix $\boldsymbol{Y}_{R}$ and the vector of $p_{R}$ dynamic coefficients $\pi_{R}$ to rigid robots, in which case the right-hand side of (1) is given by the input motor torque $\tau$.

It can be shown that similar factorizations hold also for robots with elastic joints [10]. However, one should distinguish the case when only a measure of the link position $\boldsymbol{q}$ is available, or when both the motor and link positions $(\boldsymbol{\theta}, \boldsymbol{q})$ can be measured. In the first case, one can differentiate twice in time the link equations and use the motor equations to obtain the relation

$$
\begin{gathered}
B \boldsymbol{K}^{-1}(\boldsymbol{M} \dddot{\boldsymbol{q}}+2 \dot{\boldsymbol{M}} \dddot{\boldsymbol{q}}+\ddot{\boldsymbol{M}} \ddot{\boldsymbol{q}}+\ddot{\boldsymbol{n}}) \\
+(\boldsymbol{M}+\boldsymbol{B}) \ddot{\boldsymbol{q}}+\boldsymbol{n}+\boldsymbol{\tau}_{F}=\boldsymbol{\tau}
\end{gathered}
$$

where $\boldsymbol{n}(\boldsymbol{q}, \dot{\boldsymbol{q}})=\boldsymbol{c}(\boldsymbol{q}, \dot{\boldsymbol{q}})+\boldsymbol{g}(\boldsymbol{q})$ for compactness and dependencies have been dropped. Having eliminated the dependence for motor variables, equation (4) takes the form

$$
\boldsymbol{Y}_{E, 1}(\boldsymbol{q}, \dot{\boldsymbol{q}}, \ddot{\boldsymbol{q}}, \dddot{\boldsymbol{q}}, \dddot{\boldsymbol{q}}) \boldsymbol{\pi}_{E, 1}=\boldsymbol{\tau},
$$

with a more complex regressor $\boldsymbol{Y}_{E, 1}$, having the same number of rows of the rigid case, and a much larger vector of dynamic coefficients $\boldsymbol{\pi}_{E, 1}$. In the second case, the situation is slightly simpler. It is easy to see that eqs. (1) and (2) can be rearranged as

$$
\boldsymbol{Y}_{E, 2}(\boldsymbol{q}, \dot{\boldsymbol{q}}, \ddot{\boldsymbol{q}}, \boldsymbol{\theta}, \dot{\boldsymbol{\theta}}, \ddot{\boldsymbol{\theta}}) \boldsymbol{\pi}_{E, 2}=\left(\begin{array}{c}
\mathbf{0} \\
\boldsymbol{\tau}
\end{array}\right)
$$

where the regressor $\boldsymbol{Y}_{E, 2}$ has now twice the number of rows of the rigid case.

From either (5) or (6), one can proceed in principle to dynamic identification as in the rigid case. However, apart from the more complex regression matrices and/or the larger number of (off-line) numerical differentiations needed on the measured positions, in the case of the KUKA LWR robot we don't have access to the actual input torque $\tau$ and so these methods cannot be used.

On the other hand, the torque-controlled nature of this robot and the control architecture in Fig. 2 allow to bypass this problem, by performing a separate, reduced identification. In fact, in such a situation, the model (4) can be replaced by the link equation only

$$
\boldsymbol{M}(\boldsymbol{q}) \ddot{\boldsymbol{q}}+\boldsymbol{c}(\boldsymbol{q}, \dot{\boldsymbol{q}})+\boldsymbol{g}(\boldsymbol{q})=\tau_{J}
$$

which is formally identical to the rigid case, with the elastic torque $\tau_{J}$ measured by the joint torque sensor in place of the commanded torque $\tau$.

\section{Dynamic Modeling of the KUKA LWR}

A dynamic model of the link dynamics in the form (7) can be obtained following different approaches [4]. As a result of any of these procedures, a vector containing (nonlinear) combinations of the original robot dynamic parameters (mass, coordinates of the center of mass, elements of the link inertia matrix) can be factored out from the final dynamic equations. Only the elements of this vector of dynamic coefficients (also called base parameters [3]) will be identifiable.

We have chosen to avoid any simplification or use of a priori knowledge when setting up the assumptions for the derivation of the robot dynamic model in symbolic form, in order to perform the most complete estimation of the dynamic coefficients.

Therefore, let $m_{i}$ be the unknown mass of link $i$, for $i=$ $1, \ldots, 7$. The position of the center of mass of link $i$ with respect to the $i$ th link frame is denoted as

$$
{ }^{i} \boldsymbol{r}_{i, c i}=\left(\begin{array}{lll}
c_{i x} & c_{i y} & c_{i z}
\end{array}\right)^{T}
$$

and, similarly, its inertia tensor relative to the center of mass of link $i$ will possibly be a full (symmetric) matrix

$$
{ }^{i} \boldsymbol{I}_{\ell_{i}}=\left(\begin{array}{ccc}
I_{i x x} & I_{i x y} & I_{i x z} \\
I_{i x y} & I_{i y y} & I_{i y z} \\
I_{i x z} & I_{i y z} & I_{i z z}
\end{array}\right)
$$

for $i=1, \ldots, 7$.

The total kinetic energy $\mathcal{T}(\boldsymbol{q}, \dot{\boldsymbol{q}})$ and potential energy $\mathcal{U}(\boldsymbol{q})$ are computed from the single energy terms of each link $\ell_{i}$. For the potential energy, it is

$$
\mathcal{U}=\sum_{i=1}^{7} \mathcal{U}_{\ell_{i}}=-\sum_{i=1}^{7} m_{i} \gamma^{T} \boldsymbol{r}_{0, c i}
$$

where the gravity acceleration in the absolute reference frame (see Fig. 1, assuming that the robot base is horizontal) is

$$
\gamma=\left(\begin{array}{ccc}
0 & 0 & -g_{0}
\end{array}\right)^{T}
$$

being $g_{0}>0$ the gravity acceleration constant, and the position $\boldsymbol{r}_{0, c i}$ of the center of mass of link $i$ with respect to the reference frame is computed as

$$
\left(\begin{array}{c}
\boldsymbol{r}_{0, c i} \\
1
\end{array}\right)={ }^{0} \boldsymbol{A}_{1}\left(q_{1}\right){ }^{1} \boldsymbol{A}_{2}\left(q_{2}\right) \cdots{ }^{i-1} \boldsymbol{A}_{i}\left(q_{i}\right)\left(\begin{array}{c}
{ }^{i} \boldsymbol{r}_{i, c i} \\
1
\end{array}\right) \text {. }
$$

Each term of the kinetic energy comes from König theorem,

$$
\mathcal{T}=\sum_{i=1}^{7} \mathcal{T}_{\ell_{i}}=\frac{1}{2} \sum_{i=1}^{7}\left(m_{i}{ }^{i} \boldsymbol{v}_{c i}^{T}{ }^{i} \boldsymbol{v}_{c i}+{ }^{i} \boldsymbol{\omega}_{i}^{T}{ }^{i} \boldsymbol{I}_{\ell_{i}}{ }^{i} \boldsymbol{\omega}_{i}\right),
$$

in which ${ }^{i} \boldsymbol{v}_{c i}$ is the absolute linear velocity of the center of mass of link $i$ and ${ }^{i} \boldsymbol{\omega}_{i}$ is the absolute angular velocity of the link $i$, both expressed in the same local frame.

The computation of ${ }^{i} \boldsymbol{v}_{c i}$ and ${ }^{i} \boldsymbol{\omega}_{i}$ has been performed in symbolic form by means of the following recursive and easily implementable algorithm (Moving Frames) [1]: 
1) ${ }^{0} \boldsymbol{\omega}_{0}=\mathbf{0},{ }^{0} \boldsymbol{v}_{0}=\mathbf{0}$;

2) for $i=1, \ldots, 7$,
a) ${ }^{i-1} \boldsymbol{\omega}_{i}={ }^{i-1} \boldsymbol{\omega}_{i-1}+\dot{\boldsymbol{q}}_{i}{ }^{i-1} \boldsymbol{z}_{i-1}$
b) ${ }^{i} \boldsymbol{v}_{i}={ }^{i-1} \boldsymbol{R}_{i}^{T}\left(\boldsymbol{q}_{i}\right)\left({ }^{i-1} \boldsymbol{v}_{i-1}+{ }^{i-1} \boldsymbol{\omega}_{i} \times{ }^{i-1} \boldsymbol{r}_{i-1, i}\right)$
c) ${ }^{i} \boldsymbol{\omega}_{i}={ }^{i-1} \boldsymbol{R}_{i}^{T}\left(\boldsymbol{q}_{i}\right)^{i-1} \boldsymbol{\omega}_{i}$
d) ${ }^{i} \boldsymbol{v}_{c i}={ }^{i} \boldsymbol{v}_{i}+{ }^{i} \boldsymbol{\omega}_{i} \times{ }^{i} \boldsymbol{r}_{i, c i}$.

The inertia matrix $\boldsymbol{M}(\boldsymbol{q})$ and the gravity vector $\boldsymbol{g}(\boldsymbol{q})$ are obtained from the expression of the kinetic energy $\mathcal{T}$ and potential energy $\mathcal{U}$ by symbolic differentiation as

$$
\boldsymbol{M}(\boldsymbol{q})=\nabla_{\dot{\boldsymbol{q}}}^{2} \mathcal{T}(\boldsymbol{q}, \dot{\boldsymbol{q}}), \quad \boldsymbol{g}(\boldsymbol{q})=\nabla_{\boldsymbol{q}} \mathcal{U}(\boldsymbol{q}) .
$$

Finally, the centrifugal and Coriolis velocity vector $\boldsymbol{c}(\boldsymbol{q}, \dot{\boldsymbol{q}})$ is computed using the Christoffel's symbols as

$$
\boldsymbol{c}(\boldsymbol{q}, \dot{\boldsymbol{q}})=\boldsymbol{S}(\boldsymbol{q}, \dot{\boldsymbol{q}}) \dot{\boldsymbol{q}},
$$

where the $i$ th row $s_{i}^{T}$ of the $S$ matrix is given by

$$
\boldsymbol{s}_{i}^{T}=\frac{1}{2} \dot{\boldsymbol{q}}^{T}\left(\left(\frac{\partial \boldsymbol{m}_{i}}{\partial \boldsymbol{q}}\right)+\left(\frac{\partial \boldsymbol{m}_{i}}{\partial \boldsymbol{q}}\right)^{T}-\frac{\partial \boldsymbol{M}}{\partial q_{i}}\right),
$$

being $\boldsymbol{m}_{i}$ the $i$ th column of the inertia matrix $M$.

\section{IDENTIFICATION Procedure}

The parameters in the gravity vector (mass and coordinates of the center of mass of each link) can be arranged into a vector $\boldsymbol{\pi}_{g} \in \mathbb{R}^{p_{g}}$ of coefficients so that

$$
\boldsymbol{g}(\boldsymbol{q})=\boldsymbol{Y}_{g}(\boldsymbol{q}) \boldsymbol{\pi}_{g} .
$$

The elements of vector $\boldsymbol{\pi}_{g}$ have been determined by collecting all the single products of trigonometric functions that appear in the gravity term, and taking then their coefficients when different.

The same factorization approach can be applied also to the inertia matrix $\boldsymbol{M}(\boldsymbol{q})$, although their (symmetric) elements have to be rearranged first in vector form. Let $\tilde{\boldsymbol{m}}(\boldsymbol{q})$ be the stacked vector of all lower triangular elements of the inertia matrix (henceforth, the inertia stack). In the present case of $n=7$ joints, this is a column with $n(n+1) / 2=28$ components. Similarly to (17), we have

$$
\tilde{\boldsymbol{m}}(\boldsymbol{q})=\boldsymbol{Y}_{m}(\boldsymbol{q}) \boldsymbol{\pi}_{m},
$$

where the vector of coefficients $\boldsymbol{\pi}_{m} \in \mathbb{R}^{p_{m}}$ contains the masses, the coordinates of the centers of mass, as well as the elements of the inertia tensor of the links.

The FRI software provides the numerical evaluation of the gravity vector and the inertia matrix of the LWR at the current link position using GetCurrentGravityVector and GetCurrentMassMatrix. Moreover, the current link position, measured by the motor encoders and corrected as $\boldsymbol{q}=\boldsymbol{\theta}-\boldsymbol{K}^{-1} \boldsymbol{\tau}_{J}$ (because of the joint compliance), is returned by the call GetMeasuredJointPositions. It is then possible to collect a fair amount of data even in a static way: once the robot is brought to a desired configuration, the numerical values of joint position, gravity vector, and inertia matrix are read and stored. Since the numerical values of the gravity vector and the mass matrix only depend on the link positions (and not on velocities and acceleration), the same acquisition can be performed during a motion as well. In both cases, this procedure avoids any influence of friction or uncertainty.

Data collection is repeated for a list of $N$ different (special and/or random) configurations, under the weak condition

$$
N n \gg p_{g}+p_{m} .
$$

For a generic configuration $\boldsymbol{q}_{k}$ in the list $(1 \leq k \leq N)$, we have

$$
\overline{\boldsymbol{g}}_{k}=\overline{\boldsymbol{Y}}_{g_{k}} \boldsymbol{\pi}_{g}, \quad \overline{\boldsymbol{m}}_{k}=\overline{\boldsymbol{Y}}_{m k} \boldsymbol{\pi}_{m}
$$

where the numerical gravity vector $\overline{\boldsymbol{g}}_{k}$ and the numerical inertia stack vector $\overline{\boldsymbol{m}}_{k}$ are both retrieved from the FRI, $\overline{\boldsymbol{Y}}_{g_{k}}=\boldsymbol{Y}_{g}\left(\boldsymbol{q}_{k}\right)$, and $\overline{\boldsymbol{Y}}_{m k}=\boldsymbol{Y}_{m}\left(\boldsymbol{q}_{k}\right)$. All these relations are organized as

$$
\overline{\boldsymbol{g}}=\left(\begin{array}{c}
\overline{\boldsymbol{g}}_{1} \\
\overline{\boldsymbol{g}}_{2} \\
\vdots \\
\overline{\boldsymbol{g}}_{N}
\end{array}\right)=\left(\begin{array}{c}
\overline{\boldsymbol{Y}}_{g_{1}} \\
\overline{\boldsymbol{Y}}_{g_{2}} \\
\vdots \\
\overline{\boldsymbol{Y}}_{g_{N}}
\end{array}\right) \boldsymbol{\pi}_{g}=\overline{\overline{\boldsymbol{Y}}}_{g} \boldsymbol{\pi}_{g}
$$

and

$$
\overline{\boldsymbol{m}}=\left(\begin{array}{c}
\overline{\boldsymbol{m}}_{1} \\
\overline{\boldsymbol{m}}_{2} \\
\vdots \\
\overline{\boldsymbol{m}}_{N}
\end{array}\right)=\left(\begin{array}{c}
\overline{\boldsymbol{Y}}_{m 1} \\
\overline{\boldsymbol{Y}}_{m 2} \\
\vdots \\
\overline{\boldsymbol{Y}}_{m N}
\end{array}\right) \boldsymbol{\pi}_{m}=\overline{\boldsymbol{Y}}_{m} \boldsymbol{\pi}_{m} .
$$

At this stage, one should possibly drop linearly dependent columns of the regressors in order to reach a full (column) rank condition. Consequently, some coefficients will be grouped following the procedure suggested in [3]. A coefficient $K_{j}$ can be grouped with some other coefficients $K_{j 1}, \ldots, K_{j r}$ if the column $\boldsymbol{D}^{j}$ of a regressor (either $\overline{\overline{\boldsymbol{Y}}}_{g}$ or $\left.\overline{\bar{Y}}_{m}\right)$ is linearly dependent on $\boldsymbol{D}^{j 1}, \ldots, \boldsymbol{D}^{j r}$, or

$$
\boldsymbol{D}^{j}=t_{j 1} \boldsymbol{D}^{j 1}+\ldots+t_{j r} \boldsymbol{D}^{j r}
$$

where $t_{j k}$ are constants. In this case, the column $\boldsymbol{D}^{j}$ and the coefficient $K_{j}$ can be eliminated, while the coefficients $K_{j 1}, \ldots, K_{j r}$ will be replaced by $K_{j 1}^{R}, \ldots, K_{j r}^{R}$, where $K_{j p}^{R}=K_{j p}+t_{j p} K_{j}$, for $p=1, \ldots, r$.

Condition (19) on the number $N$ of samples is used to avoid ill-conditioning of the matrices $\overline{\overline{\boldsymbol{Y}}}_{g}$ and $\overline{\overline{\boldsymbol{Y}}}_{m}$. Equations (21) and (22) are solved using a least squares technique as

$$
\hat{\boldsymbol{\pi}}_{g}=\left(\overline{\overline{\boldsymbol{Y}}}_{g}^{T} \overline{\overline{\boldsymbol{Y}}}_{g}\right)^{-1} \overline{\overline{\boldsymbol{Y}}}_{g}^{T} \overline{\boldsymbol{g}}=\overline{\overline{\boldsymbol{Y}}}_{g}^{\#} \overline{\boldsymbol{g}}
$$

and

$$
\hat{\boldsymbol{\pi}}_{m}=\left(\overline{\overline{\boldsymbol{Y}}}_{m}^{T} \overline{\overline{\boldsymbol{Y}}}_{m}\right)^{-1} \overline{\overline{\boldsymbol{Y}}}_{m}^{T} \overline{\boldsymbol{m}}=\overline{\overline{\boldsymbol{Y}}}_{m}^{\#} \overline{\boldsymbol{m}}
$$

where $\overline{\overline{\boldsymbol{Y}}}_{g}^{\#}$ and $\overline{\overline{\boldsymbol{Y}}}_{m}^{\#}$ are the left pseudoinverse matrices of $\overline{\overline{\boldsymbol{Y}}}_{g}$ and $\frac{g}{\overline{\boldsymbol{Y}}_{m}}$, respectively.

Using (17) and (18), we obtain the estimates $\hat{\boldsymbol{g}}(\boldsymbol{q})$ and $\hat{M}(\boldsymbol{q})$. From eqs. (15) and (16), we compute also the estimate of the remaining velocity terms in the dynamic 
model (7). Finally, for a generic $(\boldsymbol{q}, \dot{\boldsymbol{q}}, \ddot{\boldsymbol{q}})$ given in symbolic or numerical form, the expression

$$
\hat{\boldsymbol{\tau}}_{J}=\hat{\boldsymbol{M}}(\boldsymbol{q}) \ddot{\boldsymbol{q}}+\hat{\boldsymbol{c}}(\boldsymbol{q}, \dot{\boldsymbol{q}})+\hat{\boldsymbol{g}}(\boldsymbol{q})
$$

provides also an estimation of the vector of the torques acting at the elastic joints.

\section{Numerical Results}

We have performed several data collections and preliminary tests on the KUKA LWR-IV+ available in our laboratory, in order to get more confidence in the proposed identification procedure. From these tests, we have drawn two basic observations:

1) Since the base of our robot is on a horizontal table, the first element of the gravity vector (the gravity acting on the vertical joint axis 1) should always be identically zero. Nonetheless, the numerical data provided by the FRI may contain some small noise/calibration errors, possibly due to the fact that the supporting table may not be perfectly horizontal. We decided to simply discard this element from the estimation procedure, reducing to 6 the number of rows in $\boldsymbol{Y}_{g}(\boldsymbol{q})$.

2) The values retrieved with GetCurrentMassMatrix for the inertia elements $M_{7,6}=M_{6,7}$ were found to be always zero, independently of the link position. Therefore, since

$$
\begin{aligned}
M_{7,6}(\boldsymbol{q})= & c_{7 y} c_{7 z} m_{7} \cos \left(q_{7}\right)-I_{7 x z} \sin \left(q_{7}\right) \\
& -I_{7 y z} \cos \left(q_{7}\right)+c_{7 x} c_{7 z} m_{7} \sin \left(q_{7}\right)=0,
\end{aligned}
$$

it must be necessarily

$$
I_{7 x z}=m_{7} c_{7 x} c_{7 z}, \quad I_{7 y z}=m_{7} c_{7 y} c_{7 z} .
$$

No column drop was found to be necessary on the regressor matrix $\overline{\bar{Y}}_{g}$, which had 12 independent columns from the beginning of its contruction. On the other hand, $\overline{\bar{Y}}_{m}$ was reduced from a matrix with 108 columns to a full-rank matrix with 74 columns, by dropping linear dependent columns (and grouping the corresponding coefficients). Using (27), the total number of coefficients are $p_{g}=12$ for the gravity vector $\boldsymbol{g}(\boldsymbol{q})$ and $p_{m}=74$ for the inertia stack vector $\tilde{\boldsymbol{m}}(\boldsymbol{q})$.

The number of joint configurations used as training set in the identification procedure was chosen to be $N=1000$. For each data sample $k \in[1, N]$ and each robot joint, a random value was extracted from a uniform distribution in the admissible joint range (the upper and lower limits are $\pm 170^{\circ}$ for joints $1,3,5$ and 7 , and $\pm 120^{\circ}$ for the remaining joints), in such a way that both $\overline{\overline{\boldsymbol{Y}}}_{g}$ and $\overline{\boldsymbol{Y}}_{m}$ were found to be full column rank. A different validation set of $N_{v}=200$ joint configurations has been chosen in a similar way.
The symbolic structure of the vector $\boldsymbol{\pi}_{g}$ of dynamic coefficients that factorize $\boldsymbol{g}(\boldsymbol{q})$ is

$$
\boldsymbol{\pi}_{g}=\left(\begin{array}{c}
c_{7 y} m_{7} \\
c_{7 x} m_{7} \\
c_{6 x} m_{6} \\
c_{6 z} m_{6}+c_{7 z} m_{7} \\
c_{5 z} m_{5}-c_{6 y} m_{6} \\
c_{5 x} m_{5} \\
c_{4 x} m_{4} \\
c_{4 y} m_{4}+c_{3 z} m_{3} \\
c_{2 x} m_{2} \\
c_{3 x} m_{3} \\
c_{5 y} m_{5}+c_{4 z} m_{4}+d_{2}\left(m_{5}+m_{6}+m_{7}\right) \\
c_{2 z} m_{2}-c_{3 y} m_{3}+d_{1}\left(m_{3}+m_{4}+m_{5}+m_{6}+m_{7}\right)
\end{array}\right) .
$$

The numerical values (expressed in $[\mathrm{m} \cdot \mathrm{kg}]$ ) resulting from the identification procedure are

$$
\hat{\boldsymbol{\pi}}_{g}=\left(\begin{array}{c}
9.5457 \times 10^{-4} \\
-2.9826 \times 10^{-4} \\
8.3524 \times 10^{-4} \\
0.0286 \\
-0.0407 \\
-6.5637 \times 10^{-4} \\
1.334 \\
-0.0035 \\
-4.7258 \times 10^{-4} \\
0.0014 \\
9.4532 \times 10^{-4} \\
3.4568
\end{array}\right) .
$$

As a first validation, another identification procedure has been performed on a second, different LWR arm that was mounting on its flange a symmetric steel tip of $250 \mathrm{~g}$. The obtained result was:

$$
\hat{\pi}_{g}^{\text {tip }}=\left(\begin{array}{c}
9.5486 \times 10^{-4} \\
-2.9823 \times 10^{-4} \\
8.3534 \times 10^{-4} \\
0.0507 \\
-0.0407 \\
-6.5618 \times 10^{-4} \\
1.4320 \\
-0.0035 \\
-4.7317 \times 10^{-4} \\
0.0014 \\
9.4555 \times 10^{-4} \\
3.5580
\end{array}\right) .
$$

Note that only the values of $\hat{\boldsymbol{\pi}}_{g}(4), \hat{\boldsymbol{\pi}}_{g}(7)$, and $\hat{\boldsymbol{\pi}}_{g}(12)$ changed remarkably, while the other coefficients remained practically the same. Indeed, the values of the mass and of the position of the center of mass of link 7 should change due to the added tip mass, affecting in particular the coefficients that depend on $m_{7}$ and $c_{7 z}$. Considering the known kinematic parameters $d_{1}=0.4 \mathrm{~m}$ and $d_{2}=0.39 \mathrm{~m}$, we have computed

$$
m_{7}^{\mathrm{tip}}-m_{7}=\frac{1}{d_{1}}\left(\hat{\boldsymbol{\pi}}_{g}^{\mathrm{tip}}(12)-\hat{\boldsymbol{\pi}}_{g}(12)\right) \simeq 0.25 \mathrm{~kg}
$$

and

$$
m_{7}^{\mathrm{tip}}-m_{7}=\frac{1}{d_{2}}\left(\hat{\boldsymbol{\pi}}_{g}^{\mathrm{tip}}(7)-\hat{\boldsymbol{\pi}}_{g}(7)\right) \simeq 0.25 \mathrm{~kg},
$$


TABLE II

COMPONENTWISE MEAN SQUARE ERROR OF GRAVITY ESTIMATE RELATIVE TO THE TRAINING SET (TS) AND VALIDATION SET (VS) AND TO THE TORQUE VALIDATION EXPERIMENT (TVE)

\begin{tabular}{cccc}
\hline & TS & VS & TVE \\
\hline J1 & - & - & - \\
J2 & $9.3 \times 10^{-9}$ & $4.2 \times 10^{-7}$ & $2.1 \times 10^{-2}$ \\
J3 & $9.1 \times 10^{-10}$ & $3.9 \times 10^{-9}$ & $2.4 \times 10^{-3}$ \\
$\mathbf{J 4}$ & $3.3 \times 10^{-9}$ & $3.1 \times 10^{-8}$ & $3.3 \times 10^{-4}$ \\
$\mathbf{J 5}$ & $7.3 \times 10^{-12}$ & $7.3 \times 10^{-11}$ & $2.9 \times 10^{-6}$ \\
J6 & $6.4 \times 10^{-12}$ & $2.3 \times 10^{-10}$ & $5.8 \times 10^{-7}$ \\
J7 & $7.4 \times 10^{-13}$ & $6.02 \times 10^{-13}$ & $6.9 \times 10^{-10}$ \\
\hline
\end{tabular}

TABLE III

COMPONENTWISE MAXIMUM ABSOLUTE ERROR OF GRAVITY ESTIMATE RELATIVE TO THE TRAINING SET (TS) AND VALIDATION SET (VS) AND TO TORQUE VALIDATION EXPERIMENT (TVE)

\begin{tabular}{lccc}
\hline & TS & VS & TVE \\
\hline J1 & - & - & - \\
J2 & $6.2202 \times 10^{-4}$ & 0.0019 & 0.3707 \\
J3 & $5.3553 \times 10^{-4}$ & $1.6959 \times 10^{-4}$ & 0.1275 \\
J4 & $5.6154 \times 10^{-4}$ & $4.7839 \times 10^{-4}$ & 0.0447 \\
J5 & $2.1393 \times 10^{-5}$ & $2.3266 \times 10^{-5}$ & 0.0052 \\
J6 & $1.2362 \times 10^{-5}$ & $3.2970 \times 10^{-5}$ & 0.0031 \\
J7 & $1.5917 \times 10^{-6}$ & $2.0801 \times 10^{-6}$ & $1.0272 \times 10^{-4}$ \\
\hline
\end{tabular}

i.e., the additional mass of the tip.

The identification results for the vector $\boldsymbol{\pi}_{m}$ of dynamic coefficients that factorize $\tilde{\boldsymbol{m}}(\boldsymbol{q})$ are obtained in a similar way, but are not reported here due to lack of space However, the symbolic expressions of $\boldsymbol{\pi}_{m}$ and the numerical values of $\hat{\boldsymbol{\pi}}_{m}$ are available on the webpage http://www.diag.uniroma1.it/labrob/lwrdyn.

Using (29), we have reconstructed the gravity vector $\hat{\boldsymbol{g}}(\boldsymbol{q})$ according to (17). The first two columns of Tab. II report the mean square errors of the estimate of each component of the gravity vector $\boldsymbol{g}(\boldsymbol{q})$, respectively for the training and the validation sets. Similarly, Table III reports the maximum absolute errors.

The third column in both Tables refers to the following torque validation experiment, used to evaluate the quality of the estimation of $\boldsymbol{\pi}_{g}$ and $\boldsymbol{\pi}_{m}$. We have imposed a desired joint trajectory to the robot by means of the routine JointPositionController of the FRI library. In particular, all the joints are requested to move along the same following periodic trajectory:

$$
\boldsymbol{q}_{\mathrm{des}}(t)=\frac{\pi}{2} \cos \left(\frac{\pi}{7} t\right), \quad t \in[0, T], \quad T=14[\mathrm{~s}]
$$

so as to have a cyclic trajectory with start/end in $\boldsymbol{q}=$ $\pi / 2$ at zero velocity. We have collected then the measured joint positions and elastic torques $\tau_{J}$, see eq. (3), by means of the FRI routines GetMeasuredJointPositions and GetMeasuredJointTorques. The position data were differentiated twice numerically, in order to compute joint velocities and accelerations, and then filtered through a 4th order zero-phase digital Butterworth filter with a cutoff
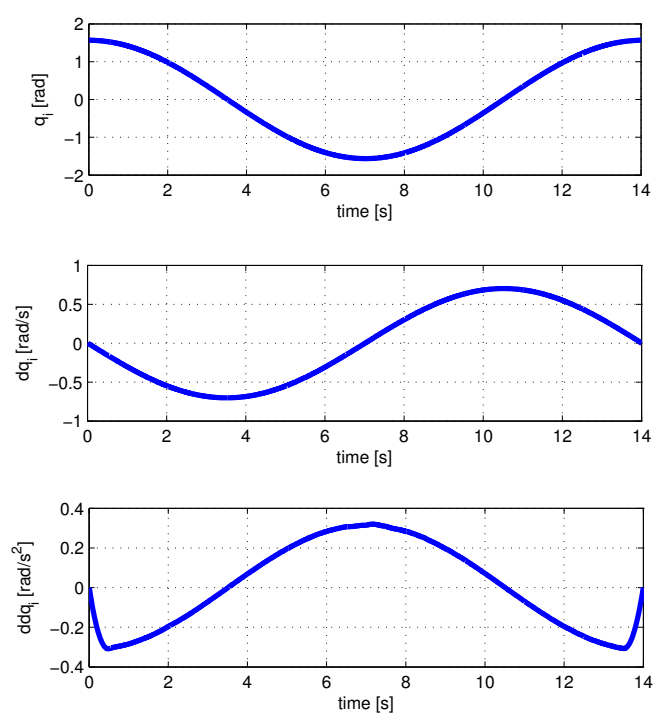

Fig. 3. Filtered joint positions (top), velocities (center) and accelerations (bottom) retrieved when imposing the same sinusoidal trajectory to all joints
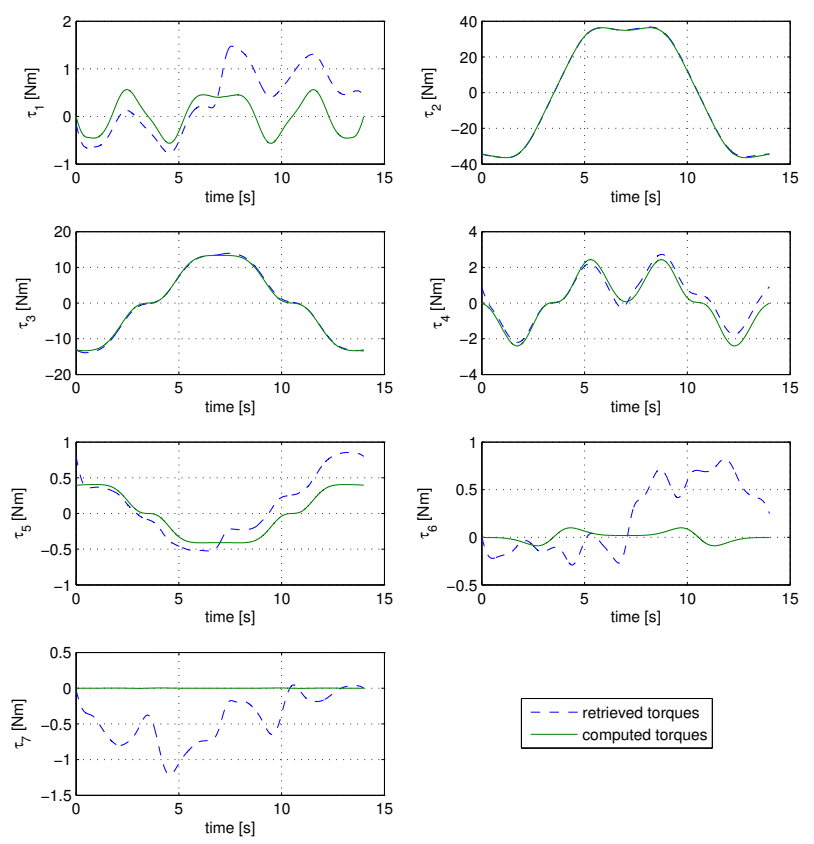

Fig. 4. Sensed joint torques $\boldsymbol{\tau}_{J}$ after filtering [dashed-blue] and estimated torques evaluated according to eq. (26) [solid-green] for the motion of Fig. 3

frequency of $1 \mathrm{~Hz}$. The obtained joint positions, velocities, and acceleration are shown in Fig. 3. They were finally substituted in eq. (26) in order to obtain the estimate $\hat{\tau}_{J}$ of the joint torques. Figure 4 shows the evolution of the filtered torque $\tau_{J}$ measured by the joint torque sensor, together with the estimated joint torque $\hat{\tau}_{J}$ for all seven robot joints.

While a good estimation was obtained for joints 2, 3, 4 , and 5, the residual errors (always smaller than $1 \mathrm{Nm}$ maximum) at the other joints 1,6 , and 7 may indicate the 


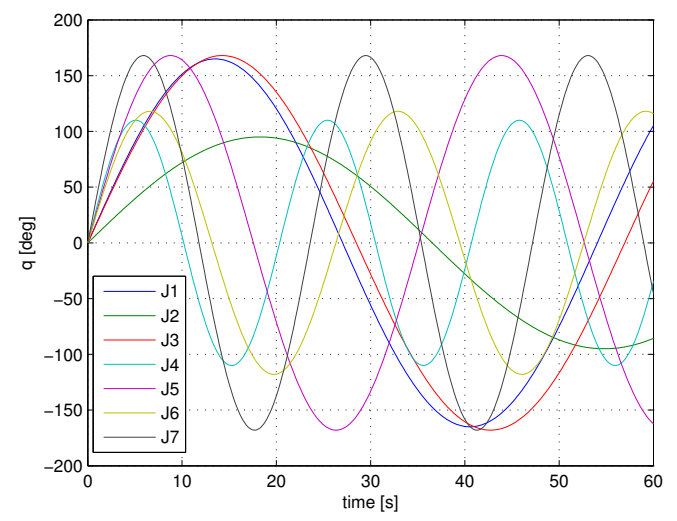

Fig. 5. Imposed joint trajectories for the final validation experiment
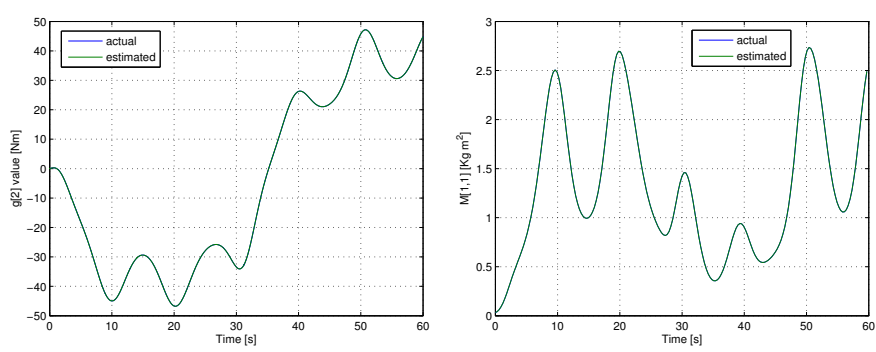

Fig. 6. Comparison between the retrieved values [blue lines] of the gravity vector element $g_{2}(\boldsymbol{q})$ (left) and of the inertia matrix element $M_{1,1}(\boldsymbol{q})$ (right) and those estimated according to the reconstructed model [green lines]

presence of unmodeled dynamics, especially static friction. For instance, although the robot executes a joint trajectory that is 'specular' around $T / 2=7 \mathrm{~s}$, the sensed torques at joints 1,5 , and 6 are considerably larger in magnitude during the return phase.

A final validation experiment has been performed in order to evaluate each of the individual components of the estimated gravity vector $\hat{\boldsymbol{g}}(\boldsymbol{q})$ and inertia matrix $\hat{\boldsymbol{M}}(\boldsymbol{q})$ (a total of $7+28=35$ elements). Sinusoidal reference trajectories of different frequency and amplitude have been imposed during 1 minute to each joint, as shown in Figure 5. The numerical values of the current link position, inertia matrix, and gravity vector have been retrieved from FRI every $120 \mathrm{~ms}$. At the end of the data acquisition, the values of each term of the estimated inertia stack $\hat{\boldsymbol{m}}(\boldsymbol{q})$ and gravity vector $\hat{\boldsymbol{g}}(\boldsymbol{q})$ are evaluated for each sample $k$, using the retrived link positions $\boldsymbol{q}(k)$. Figure 6 shows the comparison between the retrieved values of the dynamic elements $g_{2}(\boldsymbol{q})$ (gravity acting on joint 2) and $M_{1,1}(\boldsymbol{q})$ (top/left element of the link inertia matrix) and the estimated ones when 500 samples are used. This perfect agreement is confirmed also by the behavior of the other dynamic elements (see the website). We note that the element $M_{1,1}(\boldsymbol{q})$ has been chosen because it is the richest in terms of number of dynamic coefficients (it contains 52 out of the total of 74 inertial coefficients).

\section{CONCLUSIONS}

We have presented a new approach to the identification of the relevant part of the dynamic model used by the KUKA LWR robot, namely the dynamics associated to the link motion and implemented inside the KR C2 controller. The method takes advantage of the numerical values of gravity and inertia terms provided by the control software interface FRI. Unmodeled dynamic effects are not considered in this way (we cannot be more accurate or offset than the KUKA engineers) and joint elasticity is also completely masked (which was probably intentional in their control design). We need to rely on the KUKA low-level (torque or position) controller for making sure that $\boldsymbol{u}_{\text {user }}$ is actually executed. A more complete model identification that includes everything (from the input command $\tau_{\text {user }}$ to the link output position $\boldsymbol{q}$ ) should consider also the modeling of the low-level controller.

Nevertheless, the model validated so far is very promising and can help in the implementation of custom approaches, in order to define 'homemade' dynamic controllers (e.g., passivity-based tracking laws) or to reconstruct the residual vector due to collisions in an independent (and more accurate) way outside the KUKA controller. Moreover, from the obtained dynamic coefficients one can extract a set of feasible dynamic parameters of the KUKA LWR, such as link masses or inertia tensor elements, and supply realistic values to cross-platform robot simulators such as V-REP.

\section{REFERENCES}

[1] B. Siciliano, L. Sciavicco, L. Villani, and G. Oriolo, Robotics: Modeling, Planning and Control, 3rd ed. London: Springer, 2008.

[2] B. Armstrong, O. Khatib, and J. Burdick, "The explicit dynamic model and inertial parameters of the Puma 560 arm," in Proc. IEEE Int. Conf. on Robotics and Automation, 1986, pp. 510-518.

[3] W. Khalil and E. Dombre, Modeling, Identification and Control of Robots. Hermes Penton London, 2002.

[4] J. Hollerbach, W. Khalil, and M. Gautier, "Model identification," in Springer Handbook of Robotics, B. Siciliano and O. Khatib, Eds. Springer, 2008, pp. 321-344.

[5] J. Swevers, W. Verdonck, and J. De Schutter, "Dynamic model identification for industrial robots," IEEE Control Systems Mag., vol. 27, no. 5, pp. 58-71, 2007.

[6] K. Salisbury, W. Townsend, B. Eberman, and D. DiPietro, "Preliminary design of a whole-arm manipulation system (WAMS)," in Proc. IEEE Int. Conf. on Robotics and Automation, 1988, pp. 254-260.

[7] G. Hirzinger, A. Albu-Schäffer, M. Hähnle, I. Schaefer, and N. Sporer, "On a new generation of torque controlled light-weight robots," in Proc. IEEE Int. Conf. on Robotics and Automation, 2001, pp. 33563363.

[8] W. Townsend and J. Salisbury, "Mechanical design for whole-arm manipulation," in Robots and Biological Systems: Towards a New Bionics?, P. Dario, G. Sandini, and P. Aebischer, Eds. Springer, 1993, pp. 153-164.

[9] R. Bischoff, J. Kurth, G. Schreiber, R. Koeppe, A. Albu-Schäffer, A. Beyer, O. Eiberger, S. Haddadin, A. Stemmer, G. Grunwald, and G. Hirzinger, "The KUKA-DLR Lightweight Robot arm a new reference platform for robotics research and manufacturing," in Proc. 41st Int. Symp. on Robotics, 2010, pp. 741-748.

[10] A. De Luca and W. Book, "Robots with flexible elements," in Springer Handbook of Robotics, B. Siciliano and O. Khatib, Eds. Springer, 2008, pp. 287-319.

[11] KUKA.FastResearchInterface 1.0, KUKA System Technology (KST), D-86165 Augsburg, Germany, 2011, version 2.

[12] T. Kröger, "Fast Research Interface Library." [Online]. Available: http://cs.stanford.edu/people/tkr/fri/html/annotated.html

[13] M. Spong, "Modeling and control of elastic joint robots," ASME J. Dyn. Syst. Meas. Control, vol. 109, no. 4, pp. 310-319, 1987. 